\title{
Beam Diversity Analysis of Compact Microstrip Antenna with Suspended Superstrate: An Experimental Study
}

\author{
Debaprasad Barad ${ }^{1}$, and Subhrakanta Behera ${ }^{2}$ \\ ${ }^{12}$ School of Electronics Engineering, KIIT University, Bhubaneswar-24, India. \\ *corresponding author, E-mail: subhraj sp@gmail . com
}

\begin{abstract}
A multi-functional microstrip compact antenna capable of steering the main beam to eight different directions in the elevation plane is conferred in this study. The compact antenna consists of a driven patch of $28 \times 28 \mathrm{~mm}^{2}$ to bring in the resonance to $2.8 \mathrm{GHz}$, for achieving enormous application in european radar service under Wi-MAX band. The conductive layer on the superstrate deflect the beam with an angle corresponding to the position of superstrate on parasitic layer, without considering complex phase shifters and associated circuits. Proper alignment of superstrate results maximum scanning angle of $139^{\circ}$ with $59^{\circ}$ of deflection angle. The directivity of the antenna is enhanced by manipulating the parameters of the superstrate. The gain of the antenna was improved up to $\sim 6 \mathrm{dBi}$ and the efficiency is improved up to $16 \%$ using engineered superstrate. The full-wave simulation as well as analytical study was done using the IE3D EM simulator.
\end{abstract}

Keywords: Microstrip Antenna; EM Beam Manipulation; Engineered Superstrate; Gain Enhancement; Beam diversity

\section{Introduction}

In recent electromagnetic research, design of steerable antenna is frequently studied. Microstrip printed antenna (MSAs) are preferred among other type of antenna, because the MSAs are offering very high operating frequency with a more compact structure which can be fabricated easily and economically low cost [1] -[3]. Recently the application of high efficient MSAs in modern microwave communication has made the device more compact [4]. Steering the main beam of the MSAs are inevitable in recent electromagnetic studies. Steering of the main beam studied using mechanically as well as electronically beam steering. Among which electronically beam steering is well suited and somewhat easy to steer the beam with improved scanning or tilting angle [5]. The electronic beam steering has widely used, many of the wireless communication systems, especially in a mobile satellite communication and microwave radar communication [6] - [7].

The addition of high refractive surface at a suitable distance from the radiating patch has been reported for antenna radiation efficiency and enhancement of the antenna gain in [8]. Rather than this, this configuration using high refractive surface called superstrate will protect the MSAs from hazards caused by environment, when the MSAs are proposed for satellite, aircraft, missiles and other exterior communication [9]. In earlier electromagnetic research it has been demonstrated that addition of superstrate layer in $z$-plane of the microstrip patch antennas having a better steering angle instead of referring array structure [10], leaky wave model [11], phase shifter [12] and other models reported, in fact these techniques are increasing the complexity, design cost and somewhat difficult to analysis and manipulating [13].

The MSAs configured in several techniques with engineered superstrate to enhance the radiation efficiency and directivity, such as highly refractive surface, artificial magnetic superstrate, electromagnetic band gap (EBG), and some design uses dielectric slabs [14]- [16]. Use of magneto-dielectric materials having high permittivity instead of dielectric material improves the gain of the MSAs. For this operation the thickness of the superstrate should be half of the wavelength [17]. The multi-layer antenna structure is analyzed using the transverse equivalent model [18], when the source is a Hertzian dipole. The transverse equivalent network model was modified to arbitrarily orient multi-layer dielectric structure, with respect to the arbitrary feeding source. In which the antenna feeding sources are replaced by the hertzian dipoles, these Hertzain dipoles replicate the far-field properties of the designed antenna. The far-field properties are analyzed [19] $-[20]$.

This work investigates the beam steering operation of a planar antenna using an engineered superstrate. The suggested antenna operating at $2.8 \mathrm{GHz}$ has an inevitable application in European aeronautical radar service. The said Resonance with RF band ranging from 2.7-2.9 GHz massively used for sharing the spectrum with other communication service as well as between other radars. In addition to that the discussed RF band can be used for WiMAX application [21]. A high-refractive superstrate is placed at the top of the patch with suitable $\mathrm{z}$ height. The antenna directivity, gain and radiation efficiency can be enhanced by manipulating the refractive index $\left(r_{i}\right)$ of the engineered superstrate. Control on the beam deflection in $E$-Plane and $H$-Plane of the radiating electromagnetic wave is realized using this multi-layer 
dielectric-superstrate structure. In this proposed structure the superstrate is shifted along the $x-y$ plane in order to achieve the control on beam deflection. Artificial magnetic superstrate model is also investigated in this work. From the simulation study it is observed that the antenna exhibits $59^{\circ}$ of beam tilting and $139^{\circ}$ of maximum scanning angle. The directivity of the antenna is also enhanced with improved gain up to $6 \mathrm{dBi}$. The composite structure of the MSAs with engineered superstrate is analyzed in full wave electromagnetic (EM) simulation tools by Mentor graphics, which follows advance methods to analyze the iterative computational task.

\subsection{Importance of Control Beam Direction and} Improve Directivity.

Enhancing the beam directivity towards a particular direction is essential in microwave radar and satellite communication including base station. In fact increase in antenna directivity increases the sensing range of the radar which is an essential and important parameter for realizing maximum coverage. Control on radiated electromagnetic (EM) beam deflection is a novel mechanism to direct the radiated wave in the required direction with a deflection angle $\left(\theta_{t}\right)$. In steerable MSAs, higher the deflection angle $\left(\theta_{t}\right)$, higher will be the scanning angle $\left(\theta_{s}\right)$. This work is proposed for enhancing the directivity and realizing improved scanning angle $\left(\theta_{s}\right)$ [19]. The designed MSA is proposed for modern microwave radar communication as well as for satellite communication with improved gain and radiation efficiency.

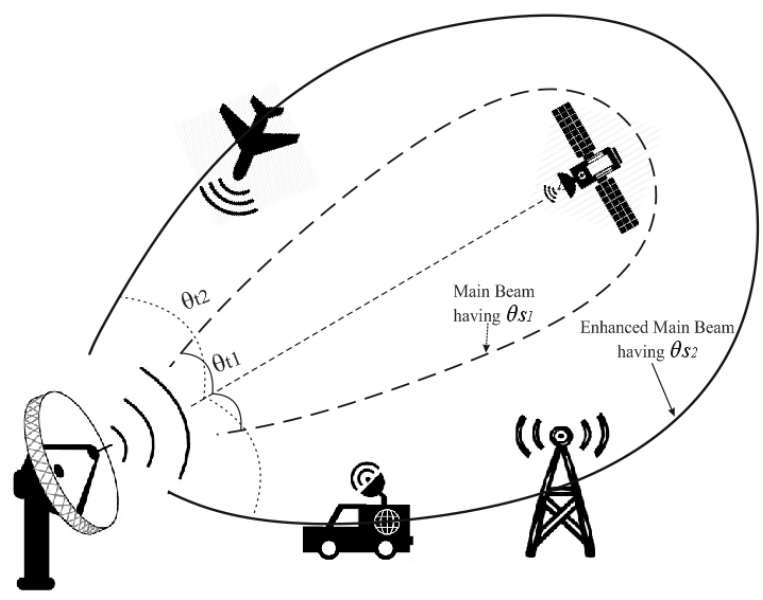

Figure 1. Enhanced performance of aeronautical Radar service using a steerable antenna.

Figure 1 illustrates the advantages of using proposed steerable antenna in base stations and microwave radar terminal, which clearly demonstrates that the steerable antenna captured maximum signals from the communication devices. In case of radar it propagates electromagnetic (EM) waves to long distance without any loss and able to cover up maximum area. Consistently the antenna having a deflection angle of $\theta_{t 1}$ which commits a scanning angle of $\theta_{s 1}$. The illustration indicates adopting of steerable antenna enhances the deflection angle $\left(\theta_{t 1}\right)$. However, this technique provides a wide range of scanning angle $\left(\theta_{s 1}\right)$ which in turn improves the coverage angle of the radar service. In the present scenario, rigorous investigation on directivity enhancement as well as improving the coverage area. Use of the proposed structure reduces the number of base station, which enables it to provide economically low cost communication and avoids the use of number of communication device in specific regions.

\section{Design and Analysis of Antenna Structure}

A novel printed antenna is suggested for beam steering with high refractive index engineered superstrate. The engineered superstrate is shifted and arranged in eight different positions above the radiator patch in $x y-$ plane along with the $E-$ Plane and $H$-Plane for realizing the maximum tilting angle. The detail antenna configuration is discussed in below section.

\subsection{Antenna design}

The proposed antenna patch is designed on an FR4 dielectric substrate having dielectric constant $\left(\varepsilon_{r}\right)$ of 3.48 , height $(h)$ of 0.762 , and loss tangent of $\tan \delta=0.0018$. The antenna configuration is like single layer, single element model. The radiator patch of the proposed antenna is placed at the top of the substrate and the ground plane is placed at the bottom of the dielectric substrate. The cross sectional view of the proposed antenna is shown in Figure 1. The radiator patch is a continuous square patch with length $\left(L_{\text {Patch }}\right)$, the physical dimension of the radiator patch is determined by using the formula given in Equ. 1 and the corresponding effective dielectric constant $\left(\varepsilon_{\text {eff. }}\right)$ is determined from Equ. 2. A $50 \Omega$ coaxial probe is connected to the patch through an SMA connector to excite the radiator patch. The feed point is initially placed at the center of the radiator patch $(x=0, y=0)$ and later the position of feeding point has been changed in order to get optimum impedance matching, gain and efficiency. From the observational study it was found that optimum result is achieved when the antenna is placed on the diagonal line with $45^{\circ}$ angle to the previous feed point, as illustrated in figure 2. The length of the proposed antenna for $f_{r}=$ $2.8 \mathrm{GHz}$ is determined by equation 1. The relationship between patch length and design frequency is derived in the section below.

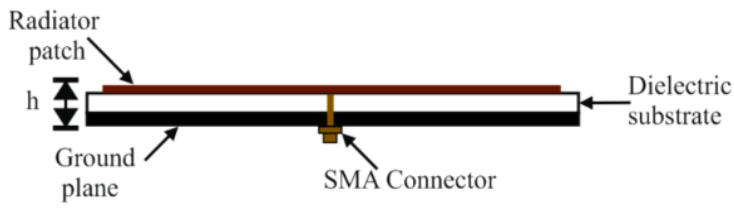

(a) Cross sectional view 


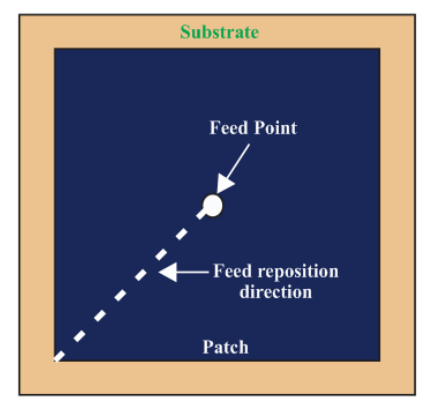

(b) Top view

Figure 2. Conventional structures of the proposed antenna.

$$
L_{\text {Patch }}=\left\{\left(\frac{1}{f_{r}}\right) \times\left(\frac{c}{\sqrt{\varepsilon_{e f f .}}}\right)\right\}
$$

The effective dielectric constant of the proposed antenna structure is determined by the specified formula [1].

\subsection{Formation of Engineered Superstrate or Magnetic superstrate}

The designed radiator patch is introduced with a high refractive engineered superstrate for beam diversity operation. The superstrate was placed at the top of the proposed antenna architecture in $Z$ - Plane with specific Electromagnetic Band Gap (EBG). The formulation of the steerable antenna structure is demonstrated in figure 3 (a). Here the square type structure is used as superstrate having dielectric constant of $\varepsilon_{s}=7$, and thickness of $h_{s}=$ $5 \mathrm{~mm}$ and physical dimension of $\left(L_{S S} \times L_{S S} \times\right.$ hs $m m 3$. An Air layer is placed in between the radiator patch and superstrate layer with layer height of $h_{a}$. The cross sectional view of the proposed steerable antenna with high refractive superstrate is shown in figure 3 (b). The position of the high refractive superstrate is repeatedly rearranged in $E$-Plane along with the xydirection for observing the characteristics of the electromagnetic wave radiation from the radiator patch. The effect of high refractive superstrate also studied by changing the superstrate parameter $\left(\varepsilon_{r}\right.$ and $\left.\mu_{r}\right)$. In fact, change in $\varepsilon_{r}$ and $\mu_{r}$ parameter gives a remarkable impact on the steering operation. By increasing the refractive index $\left(r_{i}\right)$ of the superstrate maximum scanning angle can be achieved. The details antenna parameter and its actual values are recorded in table 1.

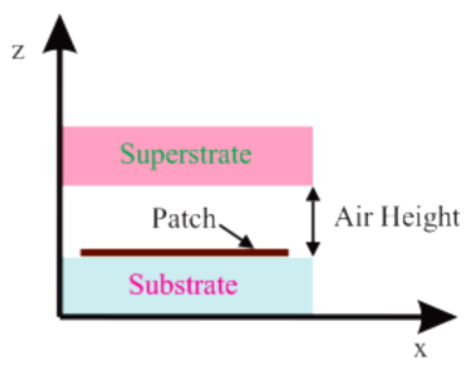

(a) Formulation of Engineered superstrate

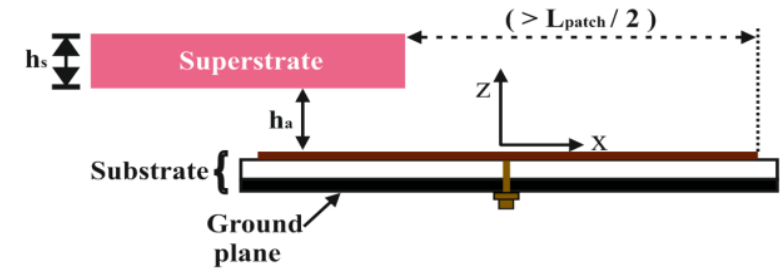

(b) Cross-view of the steerable antenna using superstrate.

Figure 3. Proposed antenna structure for Beam diversity.

Table 1: Physical dimensions of the suggested antenna.

\begin{tabular}{ll}
\multicolumn{1}{c}{ Parameters } & \multicolumn{1}{c}{ Value } \\
\hline Dielectric constant $\left(\varepsilon_{s}\right)$ & 3.48 \\
Substrate height $(\mathrm{h})$ & $0.762 \mathrm{~mm}$ \\
$\mathrm{~L}_{\text {patch }}$ & $28 \mathrm{~mm}$ \\
Dielectric constant of Superstrate $\left(\varepsilon_{r}\right)$ & 7 \\
Superstrate height $\left(h_{s}\right)$ & $5 \mathrm{~mm}$ \\
$\mathrm{~L}_{\mathrm{ss}}$ & $56 \mathrm{~mm}$ \\
Air gap $\left(\mathrm{h}_{\mathrm{a}}\right)$ & $5 \mathrm{~mm}$ \\
\hline
\end{tabular}

The Engineered superstrate is periodically shifted along the $x y$-direction to achieve beam steering operation in desired angle. However the radiated beam is deflected with an angle $\left(\theta_{t}\right)$ in the elevation plane with respect to the shifting position of the engineered superstrate meanwhile all other parameters should have to be kept as previous. This operation also yields better coverage in terms of scanning angle, the scanning angle $\left(\theta_{s}\right)$ is primarily depends on the angle of tilting $\left(\theta_{t}\right)$. In each step we have observed different beam pattern with improved $\theta_{t}$ and $\theta_{s}$.

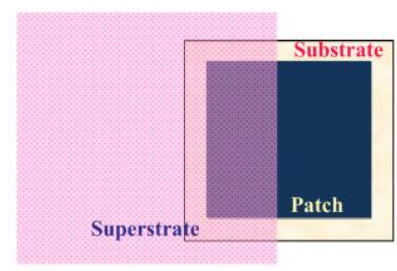

(a) Left;

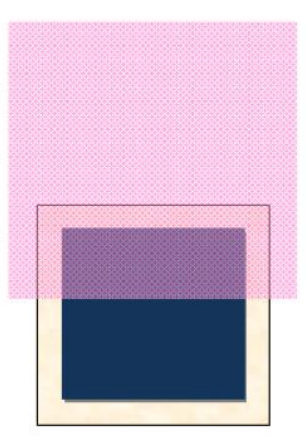

(c) Top (b) Right

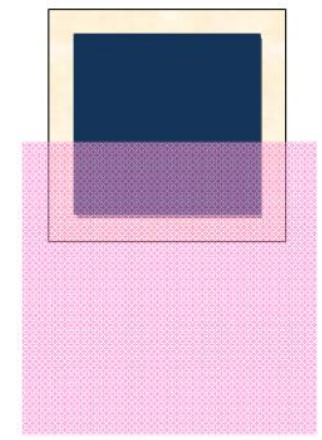

(d) Bottom

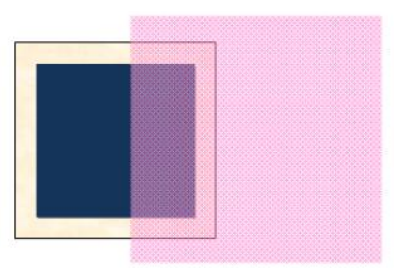

Figure 4. Manipulation of radiation pattern in different direction with superstrate at different position. 


\subsection{Antenna far field Analysis with Engineered Superstrate:}

The MSA was printed on the grounded substrate having relative permeability of $\mu_{r}$, relative permittivity of $\varepsilon_{r}$ and thickness of $h$. The superstrate above the MSA layer with a suitable distance $h_{a}$ from substrate layer in free space covers the radiating patch. The superstrate layer is having relative permeability of $\mu_{r s}$, relative permittivity of $\varepsilon_{r s}$, and layer thickness of $h_{s}$. The far-field analysis of the MSA structure can be done using several ways like, dielectric cavity model, applying reciprocity theorem, and transmission line analogy of the whole structure. In this work the far-field of proposed MSA is analyzed by the help of the transmission line model.

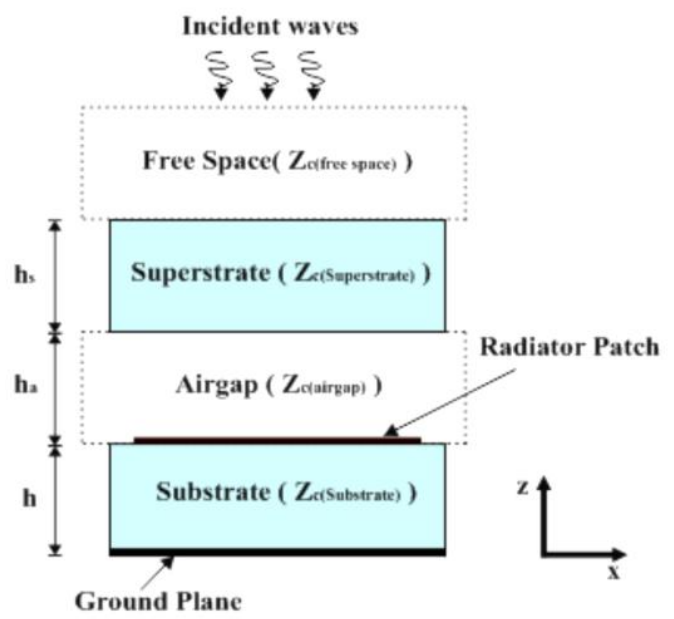

Figure 5. Equivalent Transmission line model of the suggested antenna structure (fig. 3)

The transmission line model of the proposed antenna structure with superstrate layer is shown in fig. 5. In which all the layers are replaced by its own reactance value multiple of a constant and the air layers also replaced with its reactance value multiple of a constant. The reactance values for each layer, in case of perpendicular polarization ( TE wave) is determined from the [equ. 2] - [equ. 5] and in case of parallel polarization (TM wave) is determined from [equ. 6] - [equ. 9]

Derivation of the reactance value for each layer in case of perpendicular polarization;

$$
\begin{gathered}
Z_{c(\text { substrate })}=\left(\eta_{0} \mu_{r}\right) \times\left(\frac{1}{\sqrt{\mu_{r} \varepsilon_{r}-\sin ^{2} \theta}}\right) \\
Z_{c(\text { air gap })}=\left(\eta_{0}\right) \times\left(\frac{1}{\cos \theta}\right) \\
Z_{c(\text { superstrate })}=\left(\eta_{0} \mu_{r s}\right) \times\left(\frac{1}{\sqrt{\mu_{r s} \varepsilon_{r s}-\sin ^{2} \theta}}\right) \\
Z_{c(\text { free space })}=\left(\eta_{0}\right) \times\left(\frac{1}{\cos \theta}\right)
\end{gathered}
$$

Derivation of the reactance value for each layer in case of parallel polarization;

$$
\begin{aligned}
& Z_{c(\text { substrate })}=\frac{1}{\varepsilon_{r}} \times\left(\eta_{0} \sqrt{\mu_{r} \varepsilon_{r}-\sin ^{2} \theta}\right) \\
& Z_{c(\text { air gap })}=\left(\eta_{0} \cos \theta\right) \\
& Z_{c(\text { substrate })}=\frac{1}{\varepsilon_{r s}} \times\left(\eta_{0} \sqrt{\mu_{r s} \varepsilon_{r s}-\sin ^{2} \theta}\right) \\
& Z_{c(\text { free space })}=\left(\eta_{0} \cos \theta\right)
\end{aligned}
$$

Where, $\eta_{0}=\left(\sqrt{\mu_{0} / \varepsilon_{0}}\right)$

The far-field of the designed MSA covered by the engineering superstrate is obtained by adding the field due to the radiating slots. The complete far field analysis of the steerable antenna is well investigated by Attia, et.all in [22].

\subsection{Artificial Magnetic Sources.}

Further analysis of the steerable antenna is carried out using the artificial magnetic source representation of the radiator patch. This mechanism is well studied in this section. The radiating patch of the proposed antenna is logically divided into four magnetic sources. The four magnetic source representation of the designed radiator patch is shown in figure 6. However the effect of the artificial magnetic source of the radiator patch is analyzed by representing these magnetic sources as radiating slot. The beam steering operation is observed by covering one magnetic source at a time and observing the corresponding radiation pattern. In this way the engineered superstrate is shifted above the radiator patch to cover the each magnetic source individually as shown in figure 7 and observe the corresponding beam tilting / scanning.

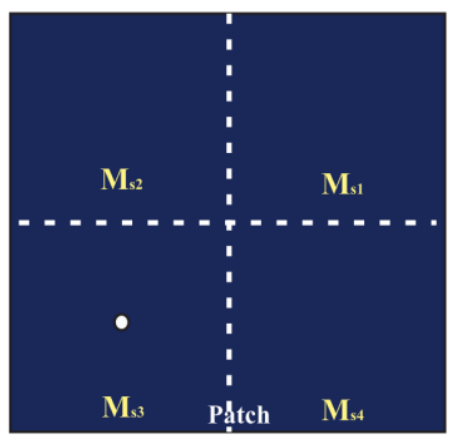

Figure 6. Analyzing the radiating patch as four magnetic sources. 
(a) Covering $\mathrm{Ms}_{1}$

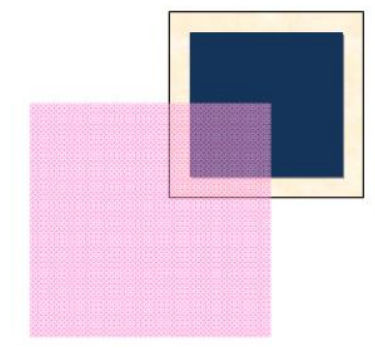

(c) Covering $\mathrm{Ms}_{3}$

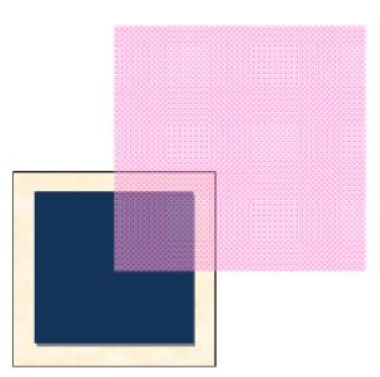

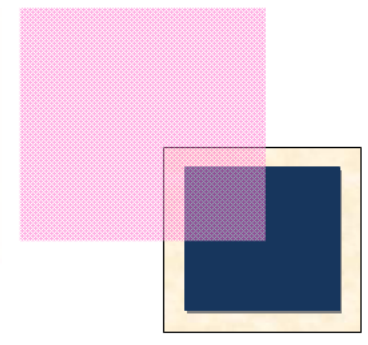

(b) Covering $\mathrm{Ms}_{2}$

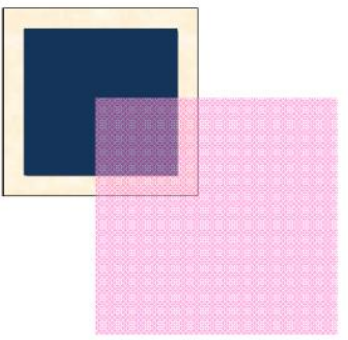

(d) Covering $\mathrm{Ms}_{4}$
Figure 7. Manipulation of radiation pattern by covering Magnetic Sources.

\subsection{Beam tilting}

The deflection angle of the main beam can be controlled in terms of changing the superstrate position over the radiator patch in $x-y$ plane. The main radiated beam can be tilted by an angle $\theta_{t}$, the tilting angle may be $+v e$ or may be $-v e$. The maximum tilting angle is due to the combined effect of superstrate parameter and its position. It has been observed that the high refractive engineered superstrate has covered only $33 \%$ area of the radiator patch for obtaining a maximum tilting angle $\left(\theta_{t}\right)$. Thereafter the high refractive superstrate is shifted along the same plane to partially cover the other parts of the radiating patch for experimental study. However the main radiated beam is steered with a desired angle by varying the high refractive index superstrate and manipulating the $r_{i}$ value. With extensively study the proposed structure is logically analyzed as four equivalent magnetic sources (as demonstrated in figure 6). Each magnetic source is covered by the high refractive superstrate and the corresponding beam patterns are captured. In fact the magnetic sources are represented as radiating slots and where the beam titling is realized due to the phase imbalance between the two radiating slots. This can be mathematically calculated from the far field contribution of each radiation slot. The transverse component of the E-field is obtained by incorporating the identical conditions on the transverse phase and electric field- vectors at the interface 1(area in between the radiator patch and superstrate) and interface 2 (Area above the superstrate layer). The steerable antenna also has capable of deflecting the main beam in both $E-$ Plane and $H-$ Plane with the proper alignment of superstrate position and manipulation of superstrate components $\left(\mu_{r s}\right.$ and $\left.\varepsilon_{r s}\right)$.

The antenna was fabricated using a different dielectric substrate of different parameter shown in fig. 8. A fixture of superstrate was done by considering a thermocol having same permittivity as air. The Moving of the superstrate position for covering different portions as discussed can be done using a simple mechtronics arrangement. Wherein Fig (a) illustrates the cross view and Fig. (b) illustrates the top view of the fabricated module. The driven patch is represented with pink color at the center of the thermocol, which evades the misalignment of the superstrate layer at the convenient position.

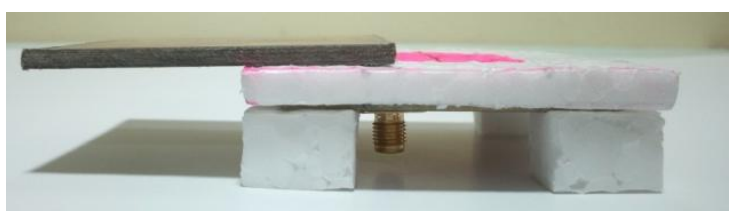

(a) Layer View

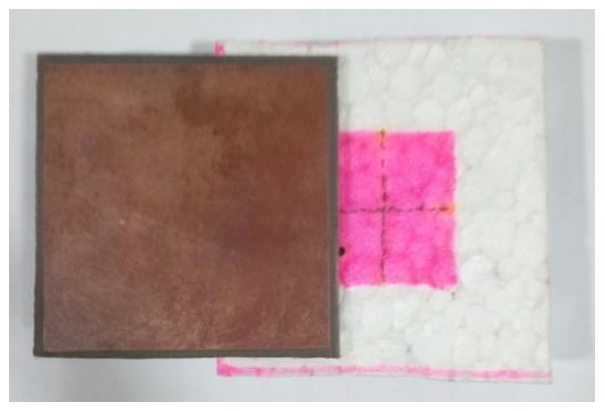

(b) Top view

Figure 8. Fabricated prototype of steerable antenna.

\section{Full Wave Analysis of Steerable Antenna}

The full wave analysis of the suggested steerable MSA is carried out using IE3D Electromagnetic simulator. The steerable antenna is optimized periodically to obtain better impedance matching as well as better gain response. The reflection co-efficient $(|\mathrm{S} 11|)$ of the proposed steerable antenna is shown in figure 9. This demonstrates that the antenna exhibits a better impedance matching at $2.8 \mathrm{GHz}$ with suitable impedance bandwidth.

Experimental study on $|\mathrm{S} 11|$ parameter identifies that the reflection co-efficient of the discussed antenna experiences a negligible change with factor 0.3 and the matching is approximately equal. It has been observed that with superstrate the antenna exhibits better matching and the frequency deviation caused by the load impedance $\left(Z_{L}\right)$ caused by the superstrate layer is somewhat negligible. However the measured $|\mathrm{S} 11|$ plot has a good agreement with the simulated $|\mathrm{S} 11|$ plot. The radiation pattern of the steerable antenna demonstrates the reformed beam, where the radiating beam is deflected from its original position with angle $\left(\theta_{t}\right)$.The $\theta_{t}$ can be calculated from the radiation pattern shown in figure 10 . However the radiating beam realizes a maximum tilting angle of $\theta_{t}=$ $59^{\circ}$ and $\theta_{s}=118^{\circ}$. The experiemental evidence by using 
both the assumptions are exposed in figure 10. It has been observed that the each step gives an approximate equal tilting angle. But somehow the gain response and radiation efficiency are optimized.

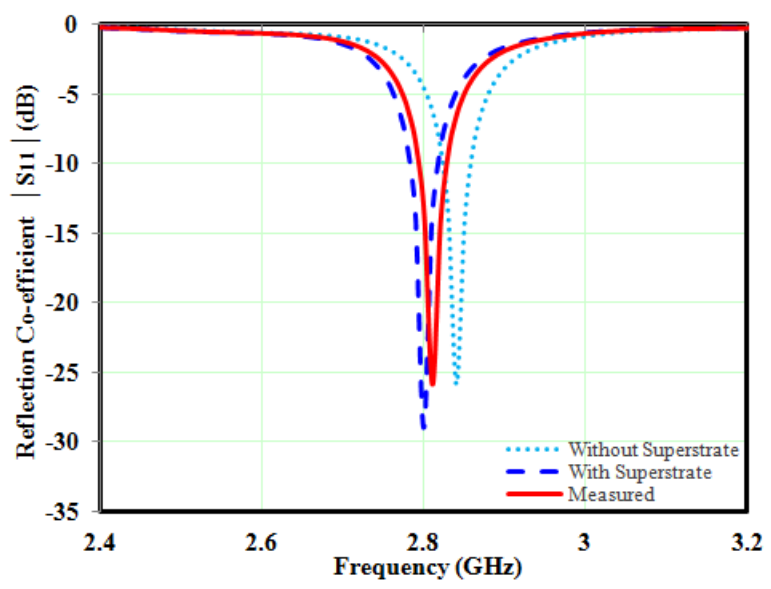

Figure 9. Return loss characteristic $\left|S_{11}\right|$ of the suggested steerable Antenna.

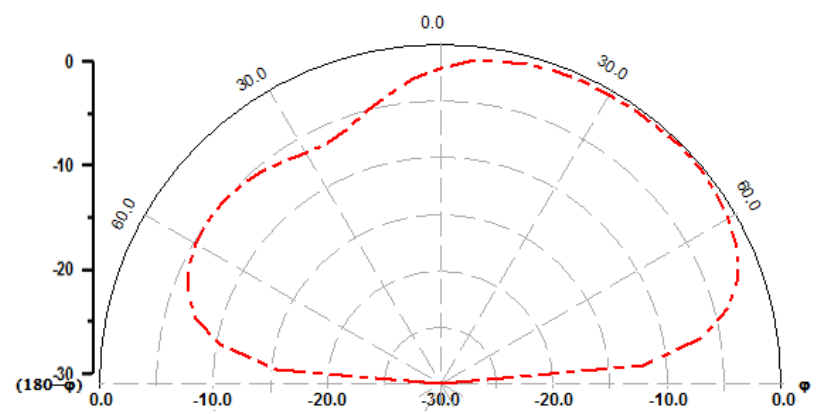

$2 \mathrm{D}$ view

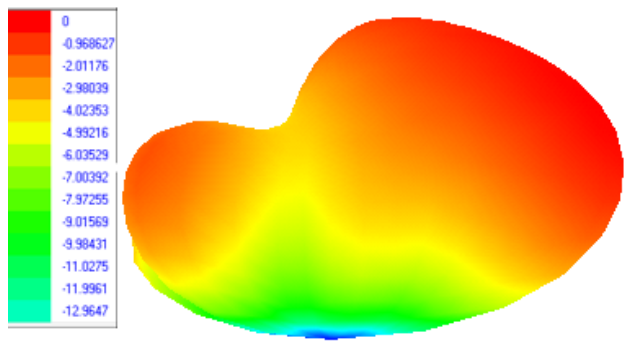

$3 \mathrm{D}$ view

(a) Radiation Pattern with covering Left Portion

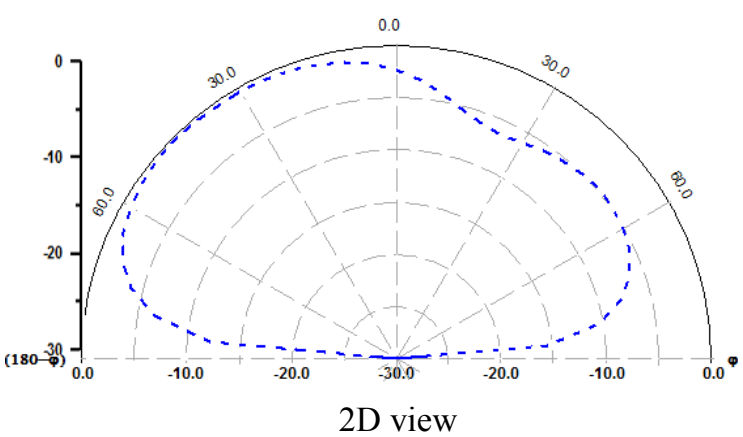

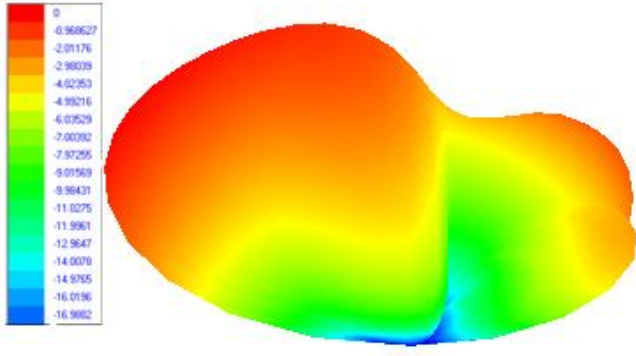

$3 \mathrm{D}$ view

(b) Radiation Pattern with covering Right Portion.
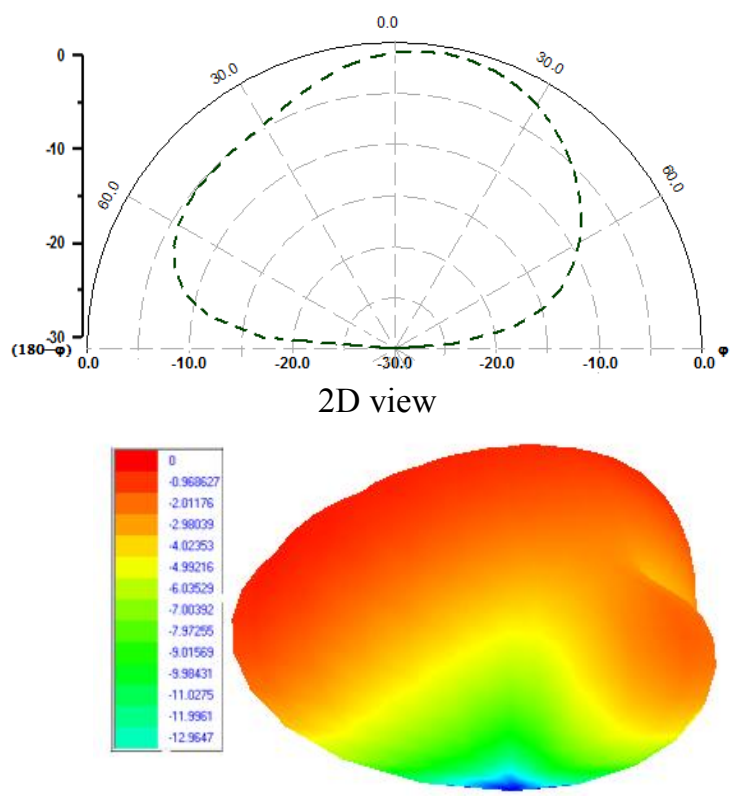

$3 \mathrm{D}$ view

(c) Radiation Pattern with covering M2.
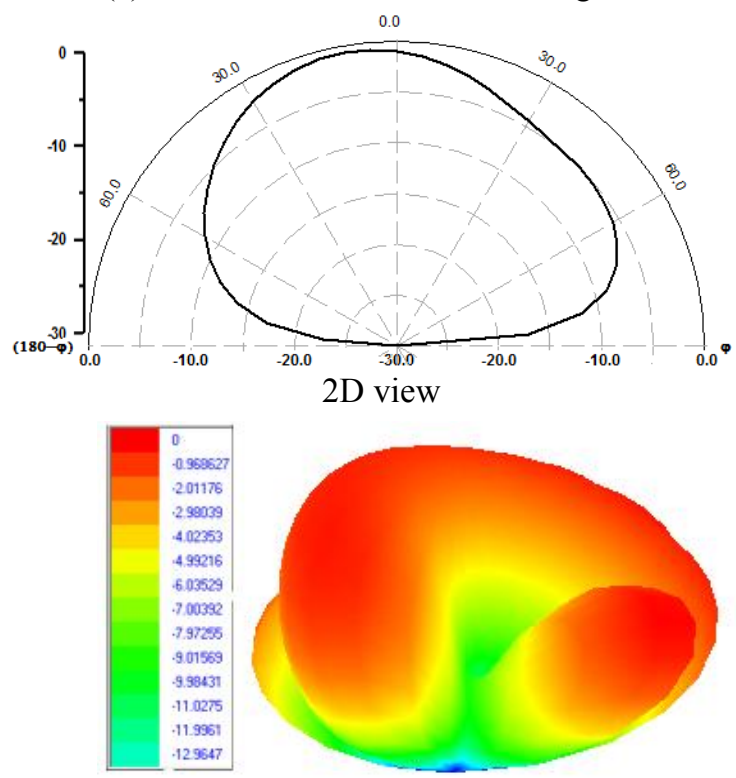

$3 \mathrm{D}$ view

(d) Radiation Pattern with covering M4.

Figure 10. Radiation pattern of the suggested steerable antenna. 
NOTE: In the radiation pattern, some beam patterns are unable to show the deflection angle due to its 2-dimensional representation. In fact, each step exhibits an improved $\theta_{t}$. It has been observed that the conductive plane at the top of the superstrate layer causes beam diversity of the radiating EM wave. The experimental consequence validates that the antenna exhibits maximum scanning angle with partially covering left side and right side. Coverage of Magnetic sources $\mathrm{M}_{2}$ and $\mathrm{M}_{4}$ provides somewhat poor deflection angle compare to left side and right side covering. Beam diversity also occurred due to partial coverage of top, bottom side, $\mathrm{M}_{1}$ and $\mathrm{M}_{3}$, but the patterns are best viewed in $360^{\circ}$ angle which is not depicted here.

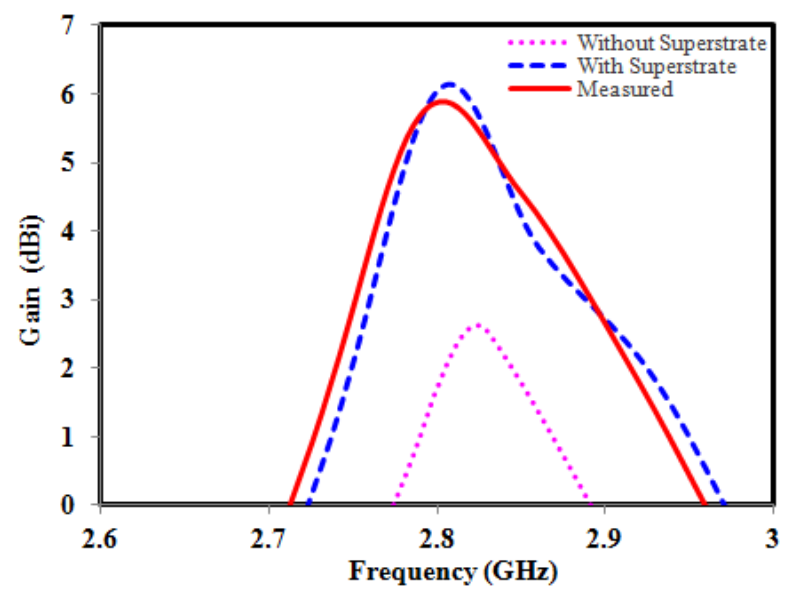

Figure 11. Gain of the suggested steerable antenna.

NOTE: Solid blue color line indicates the simulated gain response of the suggested steerable antenna and dotted red color line indicates the measured gain response.

The steerable antenna showing better gain response is plotted in figure 11. The proposed MSA has the maximum gain response of $5.5 \mathrm{dBi}$. Also the measured results are having good agreement with the simulated gain.

Table 2. Experimental Study by shifting the superstrate above the driven patch.

\begin{tabular}{ccccc}
\hline $\begin{array}{c}\text { Superstrate } \\
\text { Position }\end{array}$ & $\begin{array}{c}\text { Scanning } \\
\text { Angle }\end{array}$ & $\begin{array}{c}\text { Deflection } \\
\text { Angle }\end{array}$ & $\begin{array}{c}\text { Gain } \\
(\mathrm{dBi})\end{array}$ & $\begin{array}{c}\text { Radiation } \\
\text { Efficiency }\end{array}$ \\
\hline $\begin{array}{c}\text { Left } \\
\text { Right }\end{array}$ & $138^{\circ}$ & $58^{\circ}$ & 3.9 & $80 \%$ \\
$\begin{array}{c}\text { Top } \\
\text { Bottom }\end{array}$ & $123^{\circ}$ & $59^{\circ}$ & 3.85 & $76 \%$ \\
$\begin{array}{c}\text { Covering } \\
\text { M1 }\end{array}$ & $122^{\circ}$ & $57^{\circ}$ & 3.8 & $75 \%$ \\
$\begin{array}{c}\text { Covering } \\
\text { M2 }\end{array}$ & $105^{\circ}$ & $17^{\circ}$ & 3.82 & $76 \%$ \\
$\begin{array}{c}\text { Covering } \\
\text { M3 }\end{array}$ & $90^{\circ}$ & $27^{\circ}$ & 5.5 & $82 \%$ \\
Covering & $121^{\circ}$ & $26^{\circ}$ & 0.4 & $69 \%$ \\
M4 & & 5.2 & $81 \%$ \\
\hline
\end{tabular}

\section{Conclusions}

A novel compact MSA is designed to analyze the deflection of the radiation field and enhance the directivity using engineered superstrate. The suggested antenna has enormous application in the S-band radar communication service, more ever to European aeronautical radar service. The analysis of the antenna is based on artificial engineered superstrate and magnetic source representation of radiating patch. The direction of radiated electromagnetic wave is controlled by shifting the superstrate. The maximum tilting angle is realized by manipulating the refractive index of the engineered superstrate. For magnetic source analysis of the radiating patch give extensive observational study. In which the beam tilting is achieved in both $E$ - Plane and $H-$ Plane. The suggested patch gives a maximum tilting angle of $59^{\circ}$ at $2.8 \mathrm{GHz}$. It has been observed that this MSA structure can give maximum scanning up to $139^{\circ}$ with enhanced directivity. This is very much essential for radar, communication and satellite communication. The antenna shows maximum gain response of $6.3 \mathrm{dBi}$, which is an added advantage of this model.

\section{Acknowledgements}

This research is supported by Science and Engineering Research Board through the Department of Science and Technology, Govt. of India and school of electronics engineering, KIIT University.

\section{References}

[1] A A. balanis, "Antenna theory analysis and design", Third edition, A JOHN WILEY \& SONS, INC PUBLICATION.

[2] Ramesh Garge,"Microstrip antenna design handbook, artech house,USA.

[3] J.D.Kraus,Antennas,second edition ,M.C. Graw-Hill ,New York.

[4] M.Fallahpur,M.T. Ghasr and R.Zoughi,'Miniaturized Reconfigurable Multiband Antenna For Multiradio Wireless Communication,"in IEEE Transactions on Antennas and Propagation,vol.62,no.12,pp.6049-6059,Dec 2014.

[5] Zivin Park,Jenshan Lin "A Beam-steering Microstrip Antenna for Non-Contact Vital Sign Detection", IEEE Antennas and Wireless Propagation Letters ,vol.10,,pp.235-238,2011.

[6] Samuel C. K. Ko, and Ross D. Murch, "Compact Integrated Diversity Antenna for Wireless Communications", IEEE Transactions on Antenna and Propagation, Vol. 49, Issue No. 6, pp. 954-960, June 2001.

[7] S. Genovesi, A. Di Candia, and A. Monorchi, "Compact and Low Profile Frequency Agile Antenna for Multistandard Wireless Communication Systems", IEEE Transactions on Antenna and Propagation, Vol.62, Issue no. 3, pp. 1019-1026, March 2014.

[8] H. Y. Yang, and N. G. Alexopoulos, "Gain Enhancement Methods for Printed circuit Antennas through Multiple Superstrates", IEEE Transactions on Antennas and Propagation, Vol. AP-35, Issue. 7, pp. 860-863, July 1987.

[9] M. R. Taner, O. E. Karansan, and E. Yavuzturk, "Scheduling Beams with Different Priorities on a Military Surveillance 
Radar", IEEE Transactions on Aerospace and Electronics Systems, Vol. 48, Issue No. 2, pp. 1725-1739, April 2012.

[10] Y. Yusuf and X. Gong, "A low-cost patch antenna phased array with analog beam steering using mutual coupling and reactive loading," IEEE Antennas Wireless Propag. Lett., vol. 7, pp. 81-84, 2008.

[11] A.P. Freundorfer, M. Z. I. Bekheit, and Y. M. M. Antar, "Measurements of a Compact Surface Wave launcher Array with Application to Single frequency Beam Steering Leaky Wave Antennas", Asia-Pacific Microwave Conference, 2006.

[12] Taeksoo Ji,Hargsoon Yoon,Member, K.Abraham, Vijay K.Varadan,"Ku-Band Antenna Array Feed Distribution Network With Ferroelectric Phase Shifters on Silicon", IEEE Transactions on microwave Theory and Techniques,Vol54,no.3,March 2006.

[13] M.Faisal Abedin, and Mohammod Ali, "Effects of EBG Reflection Phase profiles on the input Impedance and Bandwidth of Ultrathin Directional Dipoles", IEEE transactions on antennas and propagation ,Vol.53,no.11,November 2005.

[14] R. Mittra, Y. Li, and K. Yoo, "A comparative study of directivity enhancement of microstriiip patch antenna with using three different superstrates", Microwave Optical Technology letter, Vol. 52, Issue no. 2, pp. 327-331, 2010.

[15] O. Siddiqui, Hussein Attia and O. M. Ramahi, \&quot;Antenna Beam Control Using High Refractive Index Superstrates"; Proceeding of the 9th International Symposium on Antenna, Propagation, and EM Theory, Guangzhou, China, Nov. 29Dec. 2, 2010.

[16] A. Foroozesh and L. Shafai, "Investigation into the effects of the patch type fss superstrate on the high-gain cavity resonance antenna design", IEEE Transaction Antenna and propagation, Vol. 58, Issue no. 2, pp. 258-270, 2010.

[17] Hussein Attia, L. Yousefi, M. M. Bait-Suwailam, M. SaidBoybay and O. M. Ramahi, "Enhanced-Gain Microstrip Antenna using Engineered Magnetic Superstrates," IEEE Antennas and Wireless Propagation Letters, vol. 8, pp. 11981201, 2009.

[18] H. Griguer, M. Drissi, E. Marzolf, H. Lalj, and F. Riouch, “ Design and characterization of a tunable DNG metamaterial superstrate for small beam steering antennas", proceeding of META'10, the 2nd International Conference on Metamaterials, Photonic Crystals and Plasmonics, pp. 255259, Cairo, Egypt, February 2010.

[19] I. Y. Hsia, "Radiation characteristics of Hertzian dipole antennas in a nonreciprocal superstrate-substrate structure" IEEE Transactions on Antennas and Propagation, Vol. 40, Issue No. 7, pp. 782-790, July 1992.

[20] Ronold W. P. King, "Electromagnetic field of dipoles and patch antennas on microstrip", RadioScience, Vol. 27, Issue No. 1, pp. 71-78, Feb. 1992.

[21] R. Womersley, "The Search for a Home for the Next Generation of Wireless Broadband", Spectrum for WiMAX in Europoe, InterConnect Communication Ltd., Merlin House, NP 16PB, UK, 2006.

[22]H. Attia, L. Yousefi, M. M. Bait-Suwailam, M. Said Boybay and O. M. Ramahi,"Analytical model for calculating the radiation field of Microstrip antenna with artificial magnetic superstrates:Theory and Experiment", IEEE transactions on antennas and propagation,vol.59, issue no ,pp.1438-1445,2011.. 